\title{
Der Staat im Neuen Testament
}

\author{
Von GÖSTA LINDESKOG
}

Jesus wurde unter Augustus geboren. Dieser, einer der grössten Staatsmänner aller Zeiten, gab wie kein zweiter der Caesaren dem antiken Staatsmythus realen Inhalt. Gemäss dem Mythus sollte ein Herrscher durch ein Reich eine neue Weltsituation schaffen. Dieses Reich sollte der tragischen Weltnot ein Ende machen. Der Reichsgründer des Mythus war mehr als ein weltlicher Politiker, er war ein Welterlöser, eine göttliche Gestalt.

Im gewaltigen Reich des Augustus herrschte die Pax Romana. Aber es gab eine unruhige Ecke, wo nicht einmal Augustus Frieden und Ruhe schaffen konnte, Palästina. Schon unter der Regierung Herodes des Grossen wurde der Nährboden für die Zeloten bereitet, die sich das Ziel setzten, wie einst die makkabäischen Freiheitskämpfer, das Fremdjoch mit Gewalt abzuschütteln ${ }^{1}$. Mit ihnen wurde der messianische Glaube zu einem politischen Faktor. Von dieser Zeit an waren die Voraussetzungen für den Freiheitskampf da, der mit der völligen äusseren Katastrophe des erwählten Volkes enden sollte.

$\mathrm{Zu}$ dem wissenschaftlich Haltbarsten der umstrittenen Jesustrilogie Ethelbert Stauffers gehört ohne Zweifel was er über das Verhältnis Roms zu Palästina schreibt ${ }^{2}$, ein Thema, das er schon in dem vielgelesenen Buch "Christus und die Caesaren" ausführlich behandelt hat ${ }^{3}$. Der Kampf zwischen Jerusalem und Rom, so wird betont, ist tiefstgesehen gegenseitig religiös bedingt: es waren zwei religiöse Mächte, die miteinander zusammenstiessen. Der römische Staat war eine göttliche Maiestas und der Kaiser

${ }^{1}$ M. Hengel, Die Zeloten. Untersuchungen zur jüdischen Freiheitsbewegung in der Zeit von Herodes I. bis 7o n. Chr. (Arbeiten zur Geschichte des Spätjudentums und Urchristentums, I), Leiden/Köln I961, S. $33^{\circ}$.

${ }^{2}$ Siehe den ersten Teil, E. Stauffer, ferusalem und Rom im Zeitalter fesu Christi (Dalp-Taschenbiicher, 33 I), Bern 1957, Kap. I-2.

${ }^{3}$ E. Stauffer, Christus und die Caesaren. Historische Skizzen, Hamburg 1960. 
war göttlich oder sogar ein Gott. Es gab im römischen Raum ein Gegenstück zu der jüdischen Messianologie ${ }^{1}$. Die Versöhnung zwischen Antonius und Octavian in Brindisi in Oktober 40 a. Chr. inspirierte Vergil zur endgültigen Ausgestaltung der ,Vierten Ekloge“, die dem römischen „Messianismus" einen grossartigen literarischen Ausdruck gab². In Aen. 6,79 I ff. grüsst Vergil Augustus als den Sohn Gottes und den, der das goldene Zeitalter bringen soll ${ }^{3}$. In Palästina huldigte Herodes der Grosse in vielfältiger Weise Augustus als einer göttlichen Offenbarung. Die Stadt Samaria taufte er zu Sebaste (von Sebastos $=$ Augustus $=$ der Anbetungswürdige) um und baute dort einen Augustus-Tempel auf. In dem von thm neu aufgebauten Caesarea (=der Kaiserstadt) an der Küste weihte er ein Templum Romae et Augusti.

Als einige Pharisäer und Herodianer (Anhänger der herodianischen Dynastie) die Frage nach dem Steuergeld an Jesus richteten, zwang er sie zuzugeben, dass sie den römischen Denar bei sich hatten (Mark. 12: 13-17 parr.). Für die Herodianer, die den Römern gegenüber Konjunkturpolitiker waren, war es wohl nicht so kompromittierend, aber um so mehr für die Pharisäer. Der römische Denar war ,ein Machtsymbol und ein Kultsym-

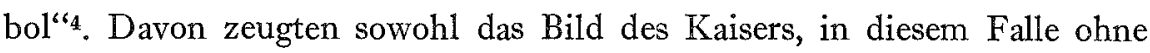
Zweifel das des Tiberius, als die Inschrift. Man konnte u. a. darauf lesen: "Tiberius Caesar Divi Augusti Filius Augustus". Auf griechisch hiess dies nach zeitgenössischen syrischen Provinzmünzen: ,,Tiberios Kaisar Theou Sebastou Hyios Sebastos" oder auf deutsch: Kaiser Tiberius, der anbetungswürdige Sohn des anbetungswürdigen Gottes (d. h. Augustus) ${ }^{5}$. So intim und alltäglich kam der Jude mit der heidnischen Okkupationsmacht und ihrer Ideologie in Berührung6.

${ }^{1}$ Stauffer, $\mathscr{F}$ erusalem, S. 24.

2 Ib.; E. Stauffer, Christus und die Caesaren, S. 84 f.

3 Stauffer, 7 erusalem, S. 27.

4 E. Stauffer, Die Botschaft fesu damals und heute (Dalp-Taschenbücher, 333), Bern I959, S. IOI.

$5 \mathrm{Ib}$.

- Die beste mir bekannte ,kontextliche" Auslegung dieser merkwürdigen Perikope hat Ethelbert Stauffer geleistet, Christus und die Caesaren, S. ז2x ff., Die Botschaft fosu, S. $98 \mathrm{ff}$. - Von den Zeloten wird erzählt, dass sie nie eine Münze bei sich haben wollten, weil es nicht erlaubt war, ein Bild bei sich zu haben oder anzusehen oder zu machen, S. G. F. Brandon, fesus and the Zealots. A Study of the Political Factor in Primitive Christianity, Manchester 1967, S. 45. 
Das Verhältnis einer Okkupationsmacht zu einem unterjochten Volk muss wohl immer spannungsvoll sein. Im Falle Judäa und Rom war es explosiv, vulkanisch. Besonders gilt dies von dem Zeitabschnitt 6-7o p. Chro, eben in derselben Periode, wo die von der Botschaft Jesu ausgehende Bewegung entstand. Dass Judäa das Joch einer fremden und eo ipso heidnischen Macht trug, war nichts weniger als eine Anomalie. Zwar war diese Anomalie nun eine alte Geschichte. Die kurze Freiheit der Makkabäerzeit war eine Episode eines, wie es schien, dauerhaften Zustandes. Die Juden hatten gelernt, unter fremden Herren zu leben und sich einen, sogar aus religiösem Gesichtspunkt sinnvollen modus vivendi zu schaffen. Aber unter den Römern traten Verhältnisse ein, die an die Zeit der Seleukiden erinnerten. Im Jahre 6 p. Chr. kamen die Juden direkt unter römische Verwaltung. Damit machte das Heidentum seinen Einzug in das Land und drohte der Heiligkeit des Landes mit Verunreinigung. Man muss die exklusive religiöse Sensibilität der Juden richtig einschätzen um ihre Reaktion zu verstehen.

Zu den unmittelbaren Veränderungen im Leben des Volkes nach der Umwandlung Judäas in ein kaiserliches Territorium gehörte die von dem neuen Statthalter Syriens Quirinius durchgeführte erste Steuereinschätzung, der Census. Dieser wurde als eine Vergewaltigung religiöser Art empfunden. Der Census ,struck at the very roots of Yahweh's sovereignty over Israelthe holy land of his ancient promise was now regarded as the property of the Roman emperor" " Jetzt tritt die zelotische Bewegung, von Judas dem Galiläer organisiert, deutlich hervor. Der Zelotismus war eine durchaus religiöse Bewegung ${ }^{2}$. Die Zeloten wollten eine Theokratie aufrichten. Ihr Grundsatz war, keinen anderen als Gott „Herr“ zu nennen. Darum war der Kaiserkultus, der den Juden überhaupt ein furchtbarer Greuel war, eben in dieser Parole gefordert. Als die praktische Konsequenz wurde die Revolte gegen die heidnische Herrschaft proklamiert.

Die Zeloten genossen Sympathien in weiten Kreisen des Volkes. Die Hoffnung auf das Kommen des Messias, der das Volk und das Land von den Fremden befreien und den Thron Davids errichten sollte, wurde in

\footnotetext{
${ }^{1}$ Brandon, Fesus and the Zealots, S. 63. Vgl. Hengel, S. $85,1_{32} \mathrm{ff}$.

2 Hengel, S. I 46 .
} 
dieser Zeit immer stärker ${ }^{1}$. Aber die Nation war geteilt. Die Realpolitiker, die Herodianer, die „neuen Sadduzäer", die priesterliche Aristokratie und der Hohe Rat, sahen das Vergebliche ein, auf militärischem Weg politische Freiheit zu gewinnen. Ihre Taktik zielte darauf hin, das möglichst Beste aus der Situation zu machen und Konflikte mit der fremden Übermacht zu vermeiden. Aber es gelang ihnen nicht, die zelotischen Aktivisten zurückzuhalten. Die Verhältnisse im Lande wurden immer chaotischer und unerträglicher, auch infolge der Misswirtschaft der römischen Prokuratoren. Aufstände, Blutvergiessen, Pogrome gehörten fast die ganze Zeit hindurch zur Tagesordnung. Die Juden hatten guten Grund, das Schlimmste zu befürchten. Caligula (37-4I) befahl, dass sein Bild in der Form einer goldenen Kolossalstatue von Zeus im Tempel aufgestellt werden sollte. Nur der Tod des Kaisers konnte die Ausführung des wahnsinnigen Plans verhindern. Aber was konnte nicht eintreffen? Die Stimmung war desperat $^{2}$.

Im Jahre $66 \mathrm{p}$. Chr. brach der grosse Aufruhr aus, der im Jahre 70 mit der Eroberung Jerusalems und der Zerstörung des Tempels endete. Die Katastrophe wurde durch die Niederwerfung des Bar-Kochba-Aufstandes im Jahre 135 endgültig. Als das grosse Schicksalssymbol figuriert jedoch in der jüdischen Erinnerung das Jahr $70^{3}$. Was damals geschah, hat auch Spuren im Neuen Testament hinterlassen und wurde für die Zukunft des werdenden Christentums entscheidend. Nach Angaben von Eusebius in seiner Kirchengeschichte (Eccl. Hist. III, v, 2-3) und Epiphanius (Adv. Haer., XXIX, 7) wanderte die judenchristliche Urgemeinde in Jerusalem vor der Katastrophe in das Ostjordanland (Pella) aus. Die Historizität der „Pellalegende“ wird von einigen bezweifelt. Uber eine Sache ist man sich jedoch einig, nämlich dass die Urgemeinde nach yo aus der Sicht verschwindet. Es fiel der heidenchristlichen Kirche ausserhalb Palästinas zu, die Lei-

${ }^{1}$ Vgl. M. Avi-Yonah, Geschichte der Fuden im Zeitalter des Talmud in den Tagen von Rom und Byzanz (Studia Yudaica. Erforschungen zur Wissenschaft des Fudentums, 2), Berlin I962, S. 9: „Der messianische Glaube wurde demnach um diese Zeit zum ersten Mal zu einem politischen Faktor von grosser Bedeutung."

${ }^{2}$ Hengel, S. I Io.

s Vgl. hierzu H.-J. Schoeps, ,Die Tempelzerstörung des Jahres 70 in der jüdischen Religionsgeschichte", Aus frïhchristlicher Zeit, I950, S. $144 \mathrm{ff}$.

4 So z. B. S. G. F. Brandon, The Fall of Ferusalem and the Christian Church, London 1957, S. 167 fr.; Brandon, Ÿesus and the Zealots, S. 208 ff. 
tung zu übernehmen, und damit entstand eine neue Situation für das Christentum im Verhältnis zum Judentum und zum römischen Staat. Innerhalb der Kirche bildete sich auch das Dogma aus, dass das Schicksal der heiligen Stadt und des Tempels göttliches Gericht über das alte Gottesvolk sei, weil es nicht an die Messianität Jesu geglaubt, sondern ihn sogar zur Kreuzigung ausgeliefert habe. Das alte Gottesvolk habe seine Rolle ausgespielt und sei seiner Erwählung verlustig gegangen. Jetzt sei die Kirche das Volk Gottes, von dem das Alte Testament sprach. Sie sei die Civitas Dei, aber viel Märtyrerblut sollte fliessen, ehe Petrus der Herrscher der Roma Aeterna, der Hauptstadt der Caesaren, wurde.

Das Judentum ist immer überwiegend eine auf diesseitige Ziele und Aufgaben eingerichtete Religion geblieben. Darum wurde seine Stellungsnahme zu den politischen Fragen wesentlich anders begründet als die der Kirche. Das Judentum ist eine ausgeprägte Nationalreligion, an ein Volk und an ein Land gebunden. Wenn man so will, kan man hier vom Mythus des Volkes und Landes sprechen. Während der vielen Jahrhunderte fremden Joches, Unfreiheit und Zerstreuung haben die Juden nie aufgehört, auf eine messianische Zukunft zu hoffen, die Wiederherstellung des Nationalheimes und der Heimholung der Diaspora. Was im Jahre 135 geschah, leitete eine Entwicklung ein, wodurch die Diaspora in immer grösserem Umfang die jüdische Lebensform wurde'. Die Juden haben gelernt, in der Zerstreuung zu leben ohne wie andere Völker ein eigenes Land zu haben, bis das grosse Mirakel in unserer Zeit eintraf. Obwohl die Juden als Volk und als Individuen mehr Leiden als andere ausgestanden haben, obgleich sie gehasst und verfolgt wurden und Gegenstand stets wiederkehrender

${ }^{1}$ Nach dem Jahre 135 ging das Stammland der Juden, Judäa, verloren (Avi-Yonah, S. I7). Nur Galiläa blieb ,,als das Hauptbollwerk des palästinischen Judentums bestehen" (Avi-Yonah, S. ı 8). Die Römer waren aber Realpolitiker. Demgemäss gewannen die Juden ,,nach dem Bar-Kochba-Krieg und der hadrianischen Verfolgung die Religions- und Lehrfreiheit, ein bedeutendes Mass von gerichtlicher Selbständigkeit, eine autonome innere Organisation und die Aufrichtung des Patriarchats als anerkannte zentrale Autorität zurück" (Avi-Yonah, S. 84). Als die Perser im Jahre 6I4 Palästina eroberten, übergaben sie den Juden Jerusalem. Aber nach drei Jahren beschloss der Perserkönig, Jerusalem als eine christliche Stadt wiederaufzubauen und die Juden zu vertreiben. Avi-Yonah beurteilt diesen Fehlschlag der jüdischen Hoffnungen folgendermassen: „Mangels eines politischen Zieles wurde das palästinensische Judentum zu einer Gruppe, die den Charakter einer Diasporagemeinde trug" (Avi-Yonah, S. 272). 
Austilgungsversuche waren, von denen der letzte der schrecklichste war, haben sie nie aufgehört zu glauben, dass die Welt verbessert werden kann und dass sie berufen sind, durch positives Handeln und Eingreifen in das weltliche Leben die Mitarbeiter Gottes zu sein um auf Erden sein Reich zu errichten. Denn das Judentum hat auch eine universale Perspektive.

Hier besteht ein Unterschied zwischen dem Judentum und dem Christentum. Zwar hat sich die Kirche im Laufe der Zeiten sehr viel mit der Welt beschäftigt. Die erste grosse Demonstration dieser Weltbezogenheit war die römische Staatskirche. Aber es gab immer Strömungen, welche gegen die Verweltlichung kämpften und zwar im richtigen Empinden, dass zum ursprünglichen Christentum eine unweltliche Perspektive gehört. Es ist schwer auszumachen, welche Worte Jesu seine ipsissima von am reinsten aufbewahrt haben. Aber die Proklamation ,Mein Reich ist nicht von dieser Welt" (Joh. I8: 36) wird mit Sicherheit einen charakteristischen Zug der Verkündigung Jesu wiedergeben. Jesus hat die nationalen Messiaserwartungen abgelehnt, die viele seiner Zeitgenossen mit seiner Person verbanden. Seine Botschaft war nicht im geläufigen Sinne des Wortes politisch $\mathbf{1}$

Es gibt heutzutage einen überzeugenden Consensus, dass die Botschaft Jesu eschatologisch war². Hier besteht eine Kontinuität zwischen Jesus und dem Urchristentum. Paulus erwartete die Parusie in der nächsten Zukunft. Die eschatologische Naherwartung wurde in den Kreisen der jüdischen Apokalyptik gepflegt. W. R. Farmer ist ohne Zweifel im Recht, wenn er von ,apocalyptic zealotism" spricht und meint, diese habe eine wichtige Rolle im Kampf gegen die Römer gespielt ${ }^{3}$. Auch das junge Christentum hat reichlich aus der jüdischen Apokalyptik geschöpft ${ }^{4}$ aber nicht zu militanten

1 J. Gwyn Griffiths, ,The Disciple's Cross“, Nerw Testament Studies, 16, 1970, S. 362 , ist der Meinung, Jesu Verhältnis zu den Römern sei das eines unbewaffneten Widerstandes. Siehe ausführlicher Anm. 2, S. 75.

${ }^{2}$ Leider ist der Begriff ,,eschatologisch“ belastet und hätte eine Definition nötig. Siehe J. Carmignac, ,, Les dangers de l'eschatologie“", New Testannent Studies, I7, I 971 , S. 365 ff.

3 W. R. Farmer, Maccabees, Zealots and Fosephus, New York 1956, S. r96. - Ein wichtiges Dokument der ,militanten Apokalyptik" ist die Kriegsrolle (IQM) aus Qumran (Krieg der Kinder des Lichts gegen die Kinder der Finsternis), die dem Zelotismus nahe in Zeit und Inhalt kommt (Hengel, S. 20, $283 \mathrm{ff}$.). Hier wird der "heilige Krieg“" geschildert.

4 Auch aus diesem Grund wurde die Apokalyptik vom normativen nachchristlichen Judentum als häretisch abgestossen. Vgl. K. Koch, Ratlos vor der Apokalyptik, 
Zwecken, sondern im Gegenteil, um die transzendente Orientierung der Zukunftshoffnung mit Hilfe der apokalyptischen Bildersprache zu verkündigen. Diese eschatologische Ausrichtung, die im Urchristentum durch den Glauben begründet wurde, Jesus sei der Messias, der mit übernatürlicher Macht ausgerüstet zur Vollendung seiner Mission bald wiederkehren würde, hatte wichtige Konsequenzen für das Verhältnis der Christen zum Leben in der Welt. Aber hier sei Vorsicht bei Beurteilungen und Verwertungen angebracht. Die lebendige Religion kennt kaum das Gesetz der ausnahmslosen Folgerichtigkeit, und es gibt immer an der Seite der Haupttendenz Nebentendenzen und dazu noch Bedürfnisse und Forderungen, die sich im Alltagsleben geltend machen. Das Christentum hat während seiner ganzen Geschichte innerhalb seiner Grenzen asketische Richtungen auftreten sehen, bisweilen extremer, um nicht zu sagen lebensverneinender Art. Aber solche Erscheinungen können nicht als das typisch Christliche gelten. Jesus machte sich in seiner Umgebung weder durch seine Lehre noch in seinem Umgang mit den Menschen wegen Asketismus bekannt. Paulus hatte eine ausgeprägte pessimistische Auffassung vom Menschen, und er bekämpft frenetisch die Lehre, dass gute Werke den Menschen erlösen können. Dies hält ihn aber nicht davon ab, in der eindringlichsten Weise an das moralische Bewusstsein des Menschen zu appellieren. Ebenso ist es ersichtlich, dass er seine Adressaten energisch zu guten Werken auffordert.

Indessen ist es offenbar, dass eine eschatologische Einstellung das Verhältnis des Urchristentums zur weltlichen Gesellschaft bestimmte (Phil. 3: 20: „Unser Staatswesen ist im Himmel“, Eph. 2: 19: „Im irdischen Staat sind wir Beisassen"; ferner Hebr. Ir: I, Jak. I: I, I. Petr. I: I). Man könnte von der urchristlichen „Nostalgie“ sprechen: „Wir haben hier keine bleibende Stätte, sondern suchen die zukünftige." Das Zitat stammt aus dem Hebräerbrief (I3: I4), über den ein deutscher Exeget, Ernst Käsemann, ein Buch mit dem erfindungsreichen Titel „Das wandernde Gottesvolk“ ge-

Gütersloh 1970, S. 32; ,Was immer wieder grosses Erstaunen hervorrufen muss, ist der Umstand, dass sich auf dem riesigen Gebiet des rabbinischen Schrifttums keine echte Apokalypse nachweisen lässt, trotz der eschatologischen Thematik, die auch die Rabbinen intensiv pflegen. Kein einziges der uns bekannten apokalyptischen Bücher, ausser Daniel, wird je bei einem Rabbinen zitiert." $\mathrm{Zu}$ beachten sind aber die sogenannten „Erlösungsmidraschim“, z. B. der Midrasch des Elijah, der im Anfang des 7. Jh. p. Chr. abgefasst wurde. Siehe Avi-Yonah, S. 26r f. 
schrieben hat. Der Autor der nachkanonischen Schrift, die der Erste Klemensbrief genannt wird (95/96 geschrieben), fängt in folgender Weise an: „Die Gemeinde Gottes, die zu Rom in der Fremde wohnt, an die Gemeinde Gottes, die zu Korinth in der Fremde wohnt."

Das palästinische Urchristentum befindet sich auch geistig innerhalb eines jüdischen Rahmens und teilt seine Reaktion den Römern gegenüber. Doch ist ein Unterschied auffallend. Die Christen Palästinas wurden in derselben Weise wie andere Juden von den Römern behandelt und hatten soweit gemeinsame Sache mit ihren Landesleuten. Aber sie gerieten auch in Konflikt mit den Vertretern des orthodoxen Judentums. Es ist wahrscheinlich zu einer Schärfung zwischen der Synagoge und der Kirche in Zusammenhang mit dem Bar Kochba-Aufruhr gekommen, weil die Christusgläubigen sich weigerten, unter Bar Kochba zu kämpfen. Rabbi Akiba sah in ihm den Messias, aber die Christen hatten ja ihren Messias, und zwar war dieser kein militanter Heiland.

Grundsätzlich war der römische Staat für die Christen wie für die Juden die Inkarnation des heidnischen Wesens. Aber es gibt wichtige Zeugnisse, die zeigen, dass die Christen sich um Lojalität gegen den römischen Staat bemühten. Eine solche Aussage ist Röm. I3: I-7:

„Jedermann sei den vorgesetzten Obrigkeiten untertan: denn es gibt keine Obrigkeit ausser Gott, und die da sind, sind von Gott verordnet. Wer sich also der Obrigkeit widersetzt, der lehnt sich gegen Gottes Verordnung auf: die sich aber auflehnen, die werden sich selbst das Urteil zuziehen. Denn die Beamten sind kein Schrecken für die gute Tat, sondern für die böse. Du willst die Obrigkeit nicht fürchten: tue das Gute und du wirst Lob von ihr erhalten: denn sie ist dir Gottes Dienerin, (die dich) zum Guten (erzieht). Wenn du aber das Böse tust, so fürchte dich: denn sie führt nicht umsonst das Schwert: denn sie ist Gottes Dienerin, Rächerin zum Erweise des Zornes an dem, der das Böse tut. Darum ist's notwendig, ihr untertan zu sein, nicht nur um des Zornes, sondern auch um des Gewissens willen. Deshalb zahlt ihr ja auch Steuern: denn sie sind Beamte Gottes, die gerade dafür Sorge tragen. Gebet allen, was ihr ihnen schuldig seid, wem (ihr) Steuer (schuldet), die Steuer; wem Zoll, den Zoll; wem Furcht, die Furcht; wem Ehre, die Ehre."

Dies ist in der Tat ein sehr merkwürdiges Schriftstück. Paulus schrieb an 
die römische Gemeinde Ende der fünfziger Jahre. Dort sass auf dem Thron Nero. Wie kann Paulus sagen, dass die Obrigkeit von Gott ist? Eine Erklärung wäre im Gottesglauben des Paulus zu suchen, im monotheistischen Universalismus. Eine ähnliche Aussage bietet der um einige Jahrzehnte später abgefasste $\mathrm{r}$. Petrusbrief $(2: \mathrm{r} 3-\mathrm{r} 7)$. Die Christen werden aufgefordert, für die Obrigkeit zu beten (r. Tim. 2: $1-2$ ). Wie für den Kaiser gebetet wurde, zeigt uns r. Klem. 6I (in Rom um 95/96 geschrieben!). Ein Christ kann für den Kaiser beten, aber er darf kein Opfer vor dem Bild des Kaisers darbringen. Hier geht die Grenze der christlichen Lojalität. Die Märtyrerakte geben dafür reichliche Belege.

Gemäss der Apostelgeschichte hat sich Paulus auf seine römische Bürgerschaft berufen (r6: 37, 22: 25). Bei der Gerichtsverhandlung vor dem Prokurator Festus in Caesarea Maritima hat Paulus an den Kaiser appelliert (25: I I): „Civis Romanus sum. Caesarem appello!“ Ist sich Paulus dessen bewusst gewesen, dass seine Missionsarbeit unter ausgedehnten Reisen durch die Ordnung und Organisation des Imperiums möglich gemacht und erleichtert wurde? Auch als Missionar geniesst er die Pax Romana.

Die Lojalität gegen den Staat ist eine christliche Pflicht: Caesaris Caesari. Aber es gibt auch eine andere Erklärung des positiven Verhaltens der Christen zum heidnischen Staat, was wir die christliche Diplomatie nennen könnten. Man kann eine prorömische Tendenz an gewissen Stellen des Neuen Testaments nachweisen, die einer antijüdischen entspricht. Diese Tendenz ist in der Darstellung des Prozesses Jesu deutlich. Es sind die Juden, die Jesus gekreuzigt haben, sie sind die Verantwortlichen. Die Gestalt des Pilatus wird allmählich in einem immer vorteilhafteren Licht dargestellt. So Luk. 23: I4 ff.1. Überhaupt ist diese Tendenz für die lukanischen Schriften charakteristisch; sie sind auch das erste deutliche Zeichen der Enteschatologisierung des Urchristentums, seiner Historisierung und Ausrichtung auf eine Existenz in der Welt.

Die Rolle der Apokalyptik für das älteste Christentum ist in der letzten

1 Vgl. den Disput der ,Juden“" mit Pilatus, Joh. I9: I2: ,Infolgedessen suchte Pilatus ihn freizulassen. Die Juden aber schrieen: 'Wenn du den freilässt, bist du kein Freund des Kaisers. Jeder, der sich zum König macht, widersetzt sich dem Kaiser." " 
Zeit Gegenstand geschärfter Aufmerksamkeit gewesen ${ }^{1}$. Bekannt ist diese Äusserung Käsemanns: „Die Apokalyptik ist — da man die Predigt Jesu nicht eigentlich als Theologie bezeichnen kann - die Mutter aller christlichen Theologie gewesen"2. Die Apokalyptik ist eine jüdische Randerscheinung, die aber im vorchristlichen Judentum eine bedeutende Rolle spielte. Sie trägt deutliche Spuren fremden Einflusses, nicht zum mindesten aus Iran stammend. Sie wird, wie schon bemerkt wurde (S. 62, Anm. 4), allmählich im nachchristlichen Judentum als häretisch aufgefasst. Dagegen lebt die apokalyptische Literatur im jungen Christentum weiter.

Zur Apokalyptik gehört die sog. Katastrophentheorie nach der Formel „Neue Himmel und eine neue Erde“. Diese kosmische Veränderung ist eine göttliche Neuschöpfung. Die Katastrophentheorie ist klar formuliert in der jüngsten Schrift des Neuen Testaments, 2. Petr. Dort heisst es (3: ro ff.): „Es kommt der Tag des Herrn wie ein Dieb, da werden die Himmel sausend vergehen, die Elemente aber werden im Brande sich aufösen und die Erde und ihre Werke werden sich finden(? $)^{3}$. Wenn dies alles sich so auflöst, wie müsst ihr euch doch auszeichnen in heiligem Wandel und in Frömmigkeit und so die Erscheinung des Gottestages erwarten und ihm entgegeneilen, an dem die Himmel im Feuerschein sich aufösen werden und die Elemente im Brande schmelzen, (an dem) wir aber einen neuen Himmel und eine neue Erde nach seiner Verheissung erwarten, wo die Gerechtigkeit wohnt." Der Verfasser betont deutlich, er schreibe dieses im Hinblick auf solche, die an die Parusie zweifelten (2: 2-3).

${ }^{1}$ Siehe u. a. Koch, Ratlos vor der Apokalyptik, und W. G. Rollins, ,The New Testament and Apocalyptic", New Testament Studies, 17, I97I, S. 454 ff.

2 E. Käsemann, „Die Anfänge christlicher Theologie", Zeitschrift fiir Theologie und Kirche, 57, 1960, S. I80. Rollins, ,The New Testament" ,beschäftigt sich kri" tisch mit dieser These Käsemanns. Rollins' Forderung nach grösserer, nuancierteren Genauigkeit bei Verwendung des Begriffes Apokalyptik ist berechtigt. Jedoch kann ich seiner Auffassung vom Verhältnisse des Urchristentums zur jüdischen Apokalyptik nicht beipflichten. Siehe seine Schlussworte, S. 476, wo er den Gegensatz des Urchristentums zur jüdischen Apokalyptik zu stark betont. Käsemann hat viel mehr recht, als Rollins zugibt. Zum semantischen Problem der Apokalyptik siehe A. N. Wilder, „Eschatological Imagery and Earthly Circumstance“, New Testament Studies, 5, 1959, S. $229 \mathrm{ff}$.

${ }^{3}$ Der Text bei Nestle: kai ge kai ta en aute erga heurethesetai findet sich $\mathrm{u}$. $a$. in $\mathrm{P}^{72}$, Cod. Vat. und Cod. Sin. aber gibt keinen Sinn. Er ist darum Gegenstand vieler Korrekturen und Konjekturen gewesen. Die Schwierigkeit liegt im Verbum heurethesetai. Andere MSS haben dafür u. a. katakaesetai und aphanisthesontai. 
In der christlichen Frömmigkeit kommt bisweilen eine radikale Jenseitssehnsucht zum Ausdruck. In dieser Perspektive verschwindet die Erde ganz: die wahre Heimat der Seele ist der Himmel. Diese radikale Unweltlichkeit lässt sich aber kaum aus der Katastrophentheorie herleiten. Es wird ausdrücklich gesagt, dass der Kosmos (Himmel und Erde) neugeschaffen werden wird. Eine neue Erde, wo Gerechtigkeit wohnt, wird die Heimat einer neuen Menschheit werden (Off. 2I: I f.). Indessen ist es deutlich, dass ein Glaube, der diese Katastrophentheorie umfasst, für die existierenden irdischen Verhältnisse wenig Interesse aufzubringen vermag. Die Vertreter der bestehenden Ordnung können nicht mit einem positiven Engagement von der Seite derer rechnen, die sich einer solchen Untergangsstimmung hingeben.

Auch wenn man die Aussagen der Apokalyptiker in die unkontrollierbare Phantasiewelt verweist, so ist man mit ihnen nicht fertig. Es ist etwas Reales, was sie in ihrer seltsamen, oft in Einzelheiten sehr dunklen Sprache sagen wollen. Es gibt einen bestimmten Zusammenhang zwischen den apokalyptischen Visionen und Vorstellungen und der Welt und Zeit der Apokalyptiker. Nun herrscht grosse Uneinigkeit darüber, wie diese Visionen zu verstehen sind. Wir können zunächst an die zeitgeschichtliche Deutung erinnern, welche die Gestalten und Szenen der Visionen mit zeitgenössischen Personen und Ereignissen zu identifizieren versucht. Das Resultat dieser Deutung ist kurz gesagt, dass die neutestamentliche Apokalyptik mit ihrem Kryptogramm eine schauderhafte Polemik gegen den römischen Staat und seine Kaiser ausübt.

Der scharfsinnige Neutestamentler Ernst Lohmeyer, einer der vielen unserer Zeit, die wegen des Konflikts ihres Glaubens mit einer heidnischen Staatsideologie den Märtyrertod gelitten haben, schrieb I926 einen Kommentar zum Buch der Offenbarung1. Er verwarf gänzlich die zeitgeschichtliche Interpretation und behauptete, die Offenbarung solle als eine überhistorische, metaphysisch-mythologische Schilderung des Endstreites zwischen der göttlichen guten Macht und der satanischen bösen verstanden werden. Lohmeyers Interpretation machte auf viele einen starken Eindruck.

${ }^{1}$ E. Lohmeyer, Die Offenbarung des fohannes (Handbuch zum Neuen Testament, hrsg. von H. Lietzmann, I6), Tübingen I926. 
Jedoch muss gesagt werden, dass sie in ihrer konsequenten Durchführung übers Ziel schoss, auch wenn sie von Nutzen war als Korrektiv gegen den Aberglauben an die Möglichkeit, in Einzelheiten eine zeirgeschichrliche Deutung durchzuführen. Zweifelsohne ist das letzte Buch der Bibel von den zeitgenössischen Ereignissen inspiriert worden. Dies gilt von der ganzen apokalyptischen Tradition, in der es zu Hause ist. Und es sind keine abderitischen Verhältnisse, welche Johannes andeutet. Er führt uns auf die Arena der Weltpolitik. Sein Buch ist ein antirömisches Buch.

Nun gibt es Apokalyptik so ziemlich überall im Neuen Testament. Man spricht z. B. von der synoptischen Apokalypse (Mark. 3 parr.). Wir werden uns hier auf eine paulinische Stelle beschränken um dann einige Texte der Apokalypse aufzugreifen. Die paulinische Stelle ist 2. Thess. 2: 3-I2. Im ersten Thessalonikerbrief, dem ältesten der uns aufbewahrten Paulusbriefe (Anfang der fünfziger Jahre), hat Paulus die eschatologischen Probleme behandelt (ז. Thess. 4: I3-5: I I). In der Gemeinde hat man indessen auf eine von Paulus nicht abgezielte Weise reagiert. Man hat geglaubt, dass die Parusie unmittelbar bevorstehe, und daraus negative Folgerungen für das Alltagsleben und seine Aufgaben gezogen. Es heisst in 2: 1-2:

„Wir bitten euch aber, ihr Brüder, wegen der Ankunft unseres Herrn Jesu Christi und unserer Vereinigung mit ihm: lasst euch nicht schnell und unbesonnen erschüttern oder erschrecken, (wenn man kommt) mit Geistesaussprüchen, Worten oder Briefen unter Berufung auf uns, als wenn (es hiesse): gekommen ist der Tag des Hern."

Darauf folgt ein Unterricht über noch nicht eingetroffene Ereignisse, die eine notwendige Voraussetzung für das Wiederkommen des Herrn sind: Zunächst muss der Abfall (he apostasia) stattfinden, und der Mensch der Gesetzlosigkeit (ho anthropos tes anomias), der auch der Sohn des Verderbens genannt wird, hervortreten. Dieser ist der „Widersacher", der Feind (ho antikeimenos), der sich wider alles was Gott oder Heiligtum heisst, erhebt. Dieser wird seinen Sitz im Tempel nehmen und sich selber zu Gott machen. Nun gibt es aber etwas was ihn zurückhält (to katechon), so dass er nicht hervortreten kann, ehe seine Zeit gekommen ist. Das Mysterium der Gesetzlosigkeit (to mysterion tes anomias) ist indessen schon wirksam. Aber es gibt einen, der es noch zurückhält (ho katechon). Erst wenn dieser aus der Welt gebracht ist, wird der Gesetzlose (ho anomos) offenbart 
werden. Aber ihn wird der Herr (Jesus) mit dem Geist seines Mundes töten und bei seiner Ankunft (te epiphania tes parousias autou) vernichten. Die Ankunft des Gesetzlosen ist ein Werk Satans mit der vollen Kraft der Lüge und mit allem Betrug der Ungerechtigkeit für die, welche verloren gehen, weil sie nicht die Liebe der Wahrheit zu ihrer Erlösung annahmen. Deswegen sendet Gott die Macht des Wahns, so dass sie an die Lüge glauben, damit alle verurteilt werden, die nicht an die Wahrheit glaubten, sondern an der Ungerechtigkeit Gefallen fanden.

Dies ist ein charakteristisches apokalyptisches Kryptogramm. Manches erinnert uns an den Stil und die Ausdrücke der Johannesapokalypse. Der Gedankengang scheint im grossen und ganzen klar zu sein. Geburtswehen werden der Messiaszeit vorausgehen. Das ist eine jüdische Vorstellung. Das Böse wird losgelassen werden, der Kampf zwischen Christus und Antichrist beginnt und endet damit, dass der Antichrist besiegt werden wird.

Für diesen Text eigentümlich sind die Begriffe to katechon, das Zurückhaltende (oder das Fesselnde) und die maskulinische Form, ho katechon, der Zurückhaltende. Eine alte Erklärung ist, dass der neutrale Ausdruck auf das Imperium Romanum und der männliche auf den Kaiser sich bezieht. Aber dann müsste auch „,der Mensch der Gesetzlosigkeit" auf eine historische Person anspielen. So weit gekommen müssen wir zugestehen, dass uns diese Deutung grosse Schwierigkeit bereitet. In diesem Fall wird es angebracht sein, auf die Warnung Lohmeyers zu hören, die zeitgeschichtliche Methode in dieser eingehenden Ausdehnung zu benutzen. William Neil sucht sich hier auf der mythologischen Linie vorwärts ${ }^{1}$. Die Stelle soll seiner Meinung nach mit Hilfe von Off. 20 erklärt werden. Hier wird gesagt, dass Satan von einem Engel für die Zeit des Millenniums gefesselt wird. Der Hintergrund ist das aus der babylonischen und persischen Mythologie herrührende Motiv eines urzeitlichen Kampfes zwischen dem Weltschöpfer und seinem Erzfeind, der hier in der Gestalt eines Untiers hervortritt. Dieser Kampf wird am Ende der Zeiten wiederholt. Aber, so setzt Neil fort, wir haben hier eine Mischung von Geschichte und Mythologie. Antiochus zur

1 W. Neil, The Epistle of Paul to the Thessalonians (The Moffatt New Testament Commentary), London 1950. O. Betz, "Der Katechon", New Testament Studies, 9, I $96_{3}$, S. 276 ff., lehnt die mythische Erklärung des Begriffes Katechon ab: er sei von Dan. 9 und II her zu deuten (S. 287). Der Katechon ist nach Betz der Kaiser! 
Zeit Daniels und später Caligula und Nero, die die jüdischen und christlichen heiligen Werte vergewaltigten, wurden in diesem mythologischen Rahmen gezeichnet. Ehe wir weitergehen, ziemt es sich, den Ausdruck „der Mensch der Gesetzlosigkeit" näher zu untersuchen. Hier stiessen wir deutlich auf die Vorstellung des Antichrists. Der Ursprung dieser Vorstellung ist mythisch, was aus dem Motiv hervorgeht, dass das Untier gefangen gehalten wird. Katechein bedeutet nämlich auch gefesselt, gefangen halten. Der Ausdruck ho katechon, 2. Thess. 2, stammt aus dem Mythus. Also ist dieser Begriff nicht ad hoc erfunden. (Die Herleitung des Begriffes aus dem Mythus wird jetzt bezweifelt. Siehe Anm. I, S. 69.) Aber, wie schon Martin Dibelius in seinem Kommentar zur Stelle sagt ${ }^{1}$, die in 2. Thess 2: $3 \mathrm{ff}$. folgende Schilderung zeigt, dass nicht Satan selbst, sondern eine menschliche Person mit satanischer Ausrüstung, der Antichrist, angedeutet wird. Ich glaube, dass Dibelius hier auf die richtige Spur kommt, aber wir werden seine Deutung in einer mehr verallgemeinernden Weise modifizieren müssen. Der Mensch der Gesetzlosigkeit, der Antichrist, ist eine Personifikation, ein Typ, eher als ein Individuum. Diese Figur mit einer historischen Gestalt, die Paulus in seinen Gedanken eventuell hatte, zu identifizieren, ist eine unmögliche Aufgabe, was natürlich nicht die Annahme ausschliesst, dass Paulus wirklich an eine bestimmte Person dachte; wahrscheinlich ist es aber nicht. Aber dieser Antichrist vertritt die bösen Kräfte, die Menschen zu ihren Werkzeugen machen. Wir werden sowohl in 2. Thess. wie in der Off. in der Regel mit Personifikationen verallgemeinernder Art zu tun zu haben, womit bezweckt wird, an historische Individuen anzuspielen. Es geht in der Apokalyptik um traditionellen mythologischen Stoff, der jedoch durch konkrete Ereignisse und Situationen aktualisiert und neu angewandt wird. Diese Auffassung schliesst also nicht die Möglichkeit aus, dass in gewissen Schilderungen ein bestimmtes historisches Individuum deutlich hervorblickt ${ }^{2}$. Die Apokalyptik ist ein mythologischer Kommentar der Gegenwartsgeschichte ${ }^{3}$.

${ }^{1}$ M. Dibelius, Die Briefe des Apostels Paulus. II. Die neun kleinen Briefe (Handbuch zum Neuen Testament, 3), Tübingen 1913, 1937.

2 Dibelius zeigt überzeugend, dass der traditionelle mythologische Stoff unter dem Einfluss des aktuellen Geschehens verändert wird: „, Sehr charakteristisch ist die Veränderung dieser traditionellen Ziige unter dem Einfluss von Zeitereignissen. Das Motiv von der Tempelschändung wird variiert im Anschluss an das Verlangen 
In der Off. gibt es indessen Texte, die, wie sie auch gedeutet werden mögen, ohne den geringsten $Z$ weifel von einer intensiven antirömischen Stimmung zeugen. Viel Märtyrerblut ist schon vergossen worden. Sowohl Paulus als Petrus gehören zu der Schar derer, die für ihren Glauben mit dem Leben gebüsst haben. Die Ereignisse unter Nero (54-68) und Domitian (8I-96) haben den Bilderfolgen des Apokalyptikers Stoff geliefert ${ }^{4}$.

$\mathrm{Zu}$ den Visionen, die ohne Zweifel Spuren der Zeit tragen, gehören die Schilderungen vom Weibe und Drachen, Kap. $\mathrm{x} 2$, und von den beiden Untieren, Kap. 13. Babylon spielt in der folgenden Darstellung eine grosse Rolle. Dass „Babylon“ ein Deckname für Rom ist, steht ausser Zweifel ${ }^{5}$. Babylon ist die grosse Hure, mit der die Könige der Erde huren, Off. I7: I. Der Seher sieht die Frau auf einem scharlachroten Untier sitzen, „das ist bedeckt mit lästerlichen Namen, hat sieben Häupte und zehn Hörner. Und war das Weib gehüllt in Purpur und in Scharlach, geschmückt mit goldnem Schmuck und köstlichem Gestein und Perlen; und einen goldnen Kelch trägt sie in ihrer Hand, gefüllt mit Greuel und dem Unrat ihrer Hurerei, und geschrieben steht auf ihrer Stirn ein Name, ein Geheimnis: Babylon die grosse, der Huren Mutter, und des Greuels der Erde. Und trunken sah ich das Weib vom Blut der Heiligen und von dem Blut der Zeugen Jesu“" (17: $3 \mathrm{ff}$. ).

Es gibt wohl kaum eine zweite Schilderung von der Inkarnation des Bösen, die an intensivem Abscheu und grandioser Pathetik die Vision der grossen Hure, des Symbols des heidnischen Wesens, übertrifft. $\mathrm{Zu}$ der Schilderung gehört auch die Vision von dem Fall Babylons (Kap. r8).

Caligulas, dass sein Bild im Tempel aufgestellt werde" (Dibelius, Die Briefe, S. 30). Reichlicher Stoff zur Auslegung dieser hier kurz behandelten Stelle bietet der grosse Kommentar von B. Rigaux, Les Epîtres aux Thessaloniciens, Paris 1956 (xxxIr + 754 S!). - Die Echtheit des 2. Thess. ist umstritten. Ich finde aber keinen Anlass, hier auf diese Frage einzugehen.

3 Vgl. A. Strobel, ,Abfassung und Geschichtstheologie der Apokalypse nach Kap. xVII. 9-12", New Testament Studies, 10, 1964, S. $433 \mathrm{ff}$.

4 Domitian beanspruchte hemmungslos göttliche Verehrung. Er war der erste Kaiser, der in Rom sich offiziell Deus Dominus nennen liess. Seine amtlichen Schreiben leitete er mit den Worten ein: „Der Herr, unser Gott, befiehlt ...“. Dieser „Gott" machte sich überdies eines grauenvollen Terrorregiments schuldig.

${ }^{5}$ Vgl. I. Petr. 5: I3: „Der Gemeinde in Babylon ... grüsst euch ...". In der rabbinischen Literatur wird der Deckname „Edom" von Rom angewandt. Gewöhnliche Bezeichnung ist hier auch malchut reschaim = die ruchlose Regierung. Siehe weiter Hengel, S. 309, Avi-Yonah, S. I28. 
In diesem Zusammenhang feiert die militante Christologie wahrhaftige Triumphe. Der Himmel tut sich auf, und der Seher sieht einen Reiter auf einem weissen Ross:

„Er heisst 'Treu und wahrhaftig', und er richtet mit Gerechtigkeit und streitet. Die Augen sein, ein Feuerbrand, und auf seinem Haupte viele Diademe, und geschrieben einen Namen, den niemand kennt denn er allein, und ist gehüllt in ein Gewand, getaucht in Blut, und heisst sein Name: 'Das Wort Gottes'. Und die Heeresscharen in dem Himmel, sie folgten ihm auf weissen Rossen, mit weissem reinem Linnen angetan. Und aus seinem Munde bricht ein scharfes Schwert, dass er damit die Völker schlage. Und er, er wird sie weiden mit ehernem Stab, und er, er tritt die Kelter des grimmen Zornesweines Gottes des Allherrschers, und trägt auf dem Gewande und auf seinem Schenkel geschrieben einen Namen: 'König der Könige und Herr der Herren'. Und einen Engel sah ich stehen in der Sonne, und er schrie mit lauter Stimme und rief $z u$ allen Vögeln, die in Himmels Mitte fliegen: 'Hieher und sammelt euch zum grossen Mahle Gottes, dass ihr verzehrt das Fleisch der Könige und Fleisch der Obersten und Fleisch der Starken und Fleisch von Ross und Reitern und aller Fleisch, von Frei und Knecht und Klein und Gross!' Und ich sah das Tier, der Erde Könige und ihre Heere da versammelt, Krieg zu führen mit dem Reiter auf dem Rosse und mit seinem Heere. Und gefangen ward das Tier, und mit ihm der Lugprophet, der Wunder tat vor ihm, mit denen er verführte, die des Tieres Zeichen tragen, und die sich beugten seinem Bild. Lebendig wurden beide da geworfen in den Pfuhl von Feuer, der in Schwefel glüht, und die übrigen getötet von dem Schwert des Reiters auf dem Rosse, das aus seinem Munde bricht; und alle Vögel wurden satt von ihrem Fleisch“ (I9: I2-2I).

Ich glaube, dass Stauffer recht hat, wenn er die Offenbarung des Johannes „das Kampfbuch gegen den domitianischen Kaisermythos" nennt".

Der grosse Rabbi Akiba sah in Simon ben Koseba (oder: Simeon ben Kosiba) den Messias und gab ihm den Ehrennamen „Bar Kochba“, der Sohn des Sterns (j. Taan. 68b; vgl. Num. 24: I 7). Selbst nannte sich Simon „Fürst über Israel". Der messianische Freiheitskrieg, der anfangs so Grosses versprach, endete - um es noch einmal zu sagen - mit einer definitiven

\footnotetext{
${ }^{1}$ Stauffer, Christus und die Caesaren, S. 193*
} 
Katastrophe für die Juden ${ }^{1}$. Rund hundert Jahre früher hatten die Römer Jesus gekreuzigt. Auf sein Kreuz hatte Pilatus die Schrift ,Jesus von Nazareth, der König der Juden" schreiben lassen. Von Zeit zu Zeit erscheint ein Buch, worin behauptet wird, Jesus sei ein politischer Messiasprätendent, der gegen die Römer Aufruhr angestiftet habe und darum gekreuzigt worden sei2. In den Evangelien gibt es Spuren einer judenchristlichen Tradition, die das populäre jüdische Messiasideal widerspiegelt. Gemäss diesem wird der Messias den Thron Davids auferrichten und das messianische Friedens- und Glücksreich gründen ${ }^{3}$. Der Engel sagt zu Maria:

„Du wirst schwanger werden und einen Sohn gebären und sollst seinen Namen Jesus nennen. Der wird ein Grosser sein und Sohn des Höchsten genannt werden, und Gott der Herr wird ihm den Thron seines Vaters David geben, und er wird über das Haus Jakobs herrschen in Ewigkeit, und seine Herrschaft wird kein Ende haben" (Luk. I: 32 f.). Folglich ist Jesus ein ben-David, aus Davids Haus und Geschlecht. Seine Geburt findet in Bethlehem, der Stadt Davids, statt (Matth. 2: I, Luk. 2: 4 ff.). Die Weisen vom

${ }^{1}$ Hadrian verwandelte Jerusalem zu einer römischen Kolonie mit dem Namen Aelia Capitolina (Aelia nach dem kaiserlichen Familiennamen Aelius, Capitolina nach Jupiter Capitolinus). Auf dem Tempelplatz wurde ein Jupitertempel aufgeführt. Den Juden war der Zutritt zur Stadt mit Umgebungen verboten. Dieses Verbot konnte aber nicht auf die Dauer konsequent aufrechterhalten werden. Indessen war es den Juden nicht gestattet, sich in der Stadt niederzulassen und dort Besitz zu erwerben (Avi-Yonah, S. 8o f.).

2 Das bekannteste Beispiel ist die Riesenarbeit des jüdischen Gelehrten R. Eisler: Iesous Basileus Ou Basileusas. Die messianische Unabhängigkeitsbezvegung vom Auftreten Yohannes des Täufers bis zum Untergang Jakobs des Gerechten nach der neuerschlossenen Eroberung von Ferusalem des Flavius fosephus und den christlichen Quellen dargestellt, I-2 (Religionswissenschaftliche Bibliothek, 9), Heidelberg I929-30 (zusammen über $1400 \mathrm{~S}$.). Siehe G. Lindeskog, Die Fesusfrage im neuzeitlichen Fudentum. Ein Beitrag zur Geschichte der Leben-Fesu-Forschung (Arbeiten und Mitteilungen aus dem Neutestamentlichen Seminar zu Uppsala, hrsg. von A. Fridrichsen, 8), Uppsala I 938, S. I 92 ff. Ein spätes Beispiel dieser Gattung ist das Büchlein von J. Carmichael, The Death of Yesus. A new solution to the historical puzale of the Gospels (Penguin Books), Harmondsworth I963. Die Tendenz dieses seltsamen Buches geht aus einem einzigen Satz hervor: „Jesus was a national leader, one of the many who as we have seen sprang up among the Jews during their long-drawn-out subjugation by Rome“ (S. I 52).

3 Zur Frage der jüdischen Vorstellung vom Messias als dem Sohn Davids siehe unter vielen Hengel, S. 304 ff. E. Lohse, „Der König aus Davids Geschlecht. Bemerkungen zur messianischen Erwartung der Synagoge“, Abraham unser Vater, Festschrift fiir O. Michel, 1963, S. $337 \mathrm{ff}$. 
Morgenland kommen um dem neugeborenen König der Juden zu huldigen (Matth. 2: 2 ff.). Die Ankunft Jesu in Jerusalem vor der Passionswoche wird als der Einzug des Messiaskönigs in seine Stadt geschildert (Matth. 2I: I ff. parr.). Das Volk jubelt:

„Hosianna, gelobt sei, der da kommt in dem Namen des Herm! Gelobt sei das Reich unsers Vaters David, das da kommt in dem Namen des Herrn! Hosianna in der Höhe!“" (Mark. I I: 9 f. parr.). Tatsache ist, dass Jesus von seinen ersten Anhängern als Messias betrachtet wurde und dass eine messianische Bewegung die Folge seines Auftretens wurde ohne jedoch in politischer Richtung entwickelt zu werden ${ }^{1}$.

Worte Jesu, die auf eine politische Zielsetzung deuten, gibt es nicht. Das einzige, das diskutiert werden könnte, wäre das schon gestreifte: „Gebet dem Kaiser was des Kaisers ist, und Gott was Gottes ist" (Matth. 22: 2I parr.). Das erste Glied dieses Logions ist so aufgefasst worden, dass damit die untertänlichen Pflichten gegenüber der Staatsmacht eingeprägt werden. Das erste Glied des Logions hat m. a. W. seinen eigenen Wert. Diese Deutung ist möglich². In der alten Kirche haben die Christen, die römische Bürger waren, das Wort als Motiv für die Pflicht der doppelten Lojalität verstanden: Lojalität gegen den Kaiser und Gehorsam gegen Gott. Aber man kann auch den Nachdruck ganz auf das zweite Glied legen mit der Frage: „Was gehört eigentlich Gott?" Mit dieser Frage verliert das erste Glied seine Selbständigkeit ${ }^{3}$.

\footnotetext{
${ }^{1}$ Eine ganze Menge ähnlicher Aussagen könnte angeführt werden. Wenn Paulus in seinem Eingangsgruss zum Römerbrief ( $\mathrm{I}: 3$ ) sagt, dass Jesus ,geboren wurde aus Davids Samen nach (seinem) Fleische"c, so stammt diese Formulierung wahrscheinlich aus einem alten judenchristlichen Credo. - Zur Sache vgl. K. Berger, „Zum traditionsgeschichtlichen Hintergrund christologischer Hoheitstitel“, New Testament Studies, I 7, I97 I, S. 393: „Angesichts der überlieferten Eigenart des Auftretens Jesu in den Evv. ist es höchst unwahrscheinlich, dass die Gemeinde mit dem Titel Christos die Tradition national-politischer Messiaserwartung aufgegriffen habe" (siehe ferner S. 399, Anm. 4). Siehe auch C. Burger, Fesus als Davidssohn. Eine traditionsgeschichtliche Untersuchung (FRLAUNT, 98), Göttingen 1970.

${ }^{2}$ Vgl. noch einmal die spirituelle Auslegung Stauffers, Christus und die Caesaren, S. I2 I ff.; Stauffer, Die Botschaft $\mathcal{F e s u , ~ S . ~} 95 \mathrm{ff}$.

${ }^{3}$ Vgl. H. Conzelmann, ,Jesus Christus", Die Religion in Geschichte und Gegenwart, 3, 1959, S. 640: „,Dass man dem Kaiser geben solle, was des Kaisers ist und Gott, was Gottes ist, meint nicht eine Relativierung (etwa dass beide Ansprüche ein Stück weit gleichberechtigt seien): Jesus weist gerade auf die Absolutheit des Gehorsams gegen Gott." Diese Auslegung stimmt mit meiner eigenen Auffassung überein.
} 
Entscheidend für die Frage nach der Stellung Jesu zur weltlichen Gesellschaft ist die Auffassung über den Prozess ${ }^{1}$. Pilatus hat Jesus zur Kreuzigung verurteilt. Die Kreuzesaufschrift gibt an, dass Pilatus Jesus als Messiasprätendent angesehen hat. Die Frage ist aber was vor dem Eingreifen Pilatus' liegt. Nach Matth. 26: 65 und Mark. I4: 64 ist Jesus von dem Hohenpriester wegen Blasphemie (gidduf, vgl. Joh. Io: 33), Verletzung der göttlichen Majestät, angeklagt worden. Dies deutet zweifelsohne einen Ketzerprozess an. Aber die Sache liegt nicht so einfach, dass der Hohe Rat um einen Ketzer loszuwerden, diesen bei Pilatus angezeigt hat unter dem Vorwand, Jesus sei ein politischer Aufwiegler. Der Hohe Rat ist davon überzeugt gewesen, dass Jesus nicht nur aus religiösen, sondern auch aus politischen Gründen gefährlich sei und zwar, weil er, ohne selbst Politiker zu sein, unter dem Volk Hoffnungen hervorrief, dass er zum Messias erwählt $\operatorname{sei}^{2}$.

D. Flusser, Fesus in Selbstzeugnissen und Bilddokumenten, München 1968, S. 81, schreibt: „Der Ausspruch ist sicher nicht römerfreundlich, aber er zeigt auch, dass Jesus kein Anhänger des Widerstandes gegen die Römer war. Das ist schon wegen seiner Sittenlehre unmöglich." Hengel, S. 346, behauptet, die Zinsgroschenfrage wird gestellt um Jesus als Anhänger des Zelotismus festzulegen. So auch O. Cullmann, Der Staat im Neuen Testament, Tübingen 1956, S. I4. Brandon versteht die Frage im zelotischen „Kontext". Siehe Brandon, Fesus and the Zealots, S. 346; S. G. F. Brandon, The Trial of Fesus of Nazareth (Historical Trial Series), London 1968, S. 67: ,Jesus ruled decisively against the payment of tribute. Caesar could, ironically, have what was his; but the Holy Land of Judaea, and its resources, were emphatically not his but God's."

${ }^{1}$ Die Literatur vermehrt sich immerfort. Siehe u. a. J. Blinzler, Der Prozess fesu. Das jüdische und das römische Gerichtsverfahren gegen fesus auf Grund der ältesten Zeugnisse dargestellt und beurteilt, Regensburg 1960; P. Winter, On the Trial of Jesus (Studia Fudaica. Forschungen zur Wissenschaft des Fudentums, 1), Berlin 1961. (Vgl. G. Lindeskog, ,Der Prozess Jesu im jüdisch-christlichen Religionsgespräch“, Abraham unser Vater. Festschrift für O. Michel, I963, S. 325 ff.), Brandon, The Trial of Fesus.

${ }^{2}$ Brandon, The Trial of Jesus, S. 92, betont die politische Seite: ,The Jewish leaders were primarily concerned with Jesus as one who menaced the existing social and political order, and not as a religious heretic." - Die Frage nach dem ,Nationalismus" Jesu ist heutzutage auch sonst Gegenstand der Aufmerksamkeit. Cullmann, S. 7, sagt, dass Jesus von den Römern als Zelot zum Kreuzestod verurteilt wurde. So auch Hengel, S. $346 \mathrm{ff}$. „Räuber" (lestes) scheint ein Scheltwort für ,Zelot" von Seiten der Gegner gewesen zu sein (so oft bei Josephus). Bemerkenswert ist, dass diese Vokabel mehrmals in der Leidensgeschichte vorkommt: ,Jesus erlitt zusammen mit 2 'Räubern' (Mark. I5: 27 parr., Joh. I9: I 8), die vielleicht wirkliche Zeloten waren, denselben Tod wie so viele Angehörige der jüdischen Freiheitsbewegung 
Selbst hat Jesus mit aller Deutlichkeit jeden Versuch seitens des Volkes abgelehnt, ihn in ein politisch-messianisches Unternehmen zu engagie-

vor und nach ihm" (S. 347). Barabbas (Mark. I5: 7 parr.) gehörte nach Hengel (S. 247) wohl ebenfalls der zelotischen Bewegung an. Der Evangelist Johannes (I 8: 40) nennt ihn obendrin lestes. - S. G. F. Brandon hat sich besonders den mit der Frühgeschichte des Christentums zusammenhängenden politischen Problemen gewidmet (siehe seine oben angeführten Bücher The Fall of Ferusalem and the Christian Church, Fesus and the Zealots und The Trial of Jesus of Nazareth). Dabei hat er den Zeitabschnitt 6-70 p. Chr. mit grosser Gründlichkeit ermittelt. Ich fasse in aller Kürze zusammen: Die zelotische Bewegung bestimmt den Ablauf dieser Periode der jüdischen Geschichte. Jesus war kein Zelot, aber die von ihm ausgehende Bewegung läuft mit der zelotischen weithin parallel (vgl. Cullmann, S. 34, Hengel, S. 307, 345). Sein Einzug in Jerusalem und die Tempelreinigung sind von ihm bewusst durchgeführte messianische Demonstrationen. Er richtet seinen Kampf gegen die priester liche Aristokratie, aber damit wird er auch ein Insurgent gegen die Römer (Brandon, fesus and the Zealots, S. 33I ff. Vgl. aber S. 356: die Überzeugung Jesu vom baldigen Hereinbruch des Reiches Gottes machte ihn weniger interessiert für eine direkte antirömische Massnahme). Es handelt sich tatsächlich um eine militante Aktion. Das Auftreten Jesu im Tempel, das nicht das Werk eines Einzigen war, erreicht nicht sein Ziel. Jesus versteht, dass seine Sache verloren ist. Er zieht sich mit seinen Jüngern nach Gethsemane zurück. Hier wird er von einer von dem Hohenpriester (nach Joh. sogar in Zusammenarbeit mit den Römern) ausgesandten Truppe gefangen genommen. Nach einer Voruntersuchung von Hannas oder dem Synedrium wird er als messianischer Aufrührer von Pilatus verurteilt, gekreuzigt und zwar zusammen mit zwei Zeloten, die an ein em von Barabbas zur gleichen Zeit wie die Tempelreinigung geleiteten Aufruhr teilgenommen hatten(!). Von der Auferstehung Jesu überzeugt hielten seine Gläubigen zusammen in der Hoffnung, Jesus werde, mit übernatürlicher Kraft ausgerüstet, bald zurückkommen um sein messianisches Werk zu vollenden, was auch die endgültige Überwindung der Römer einschliesse (Brandon, Fesus and the Zealots, S. 218). Die Jesusgemeinde kämpft mit ihren Volksgenossen im verzweifelten Endkrieg zusammen und geht dabei unter (Brandon, The Fall of Ferusalem, S. I80). Danach entsteht als eine völlig neue Religion, deren Urheber Paulus ist, das Heidenchristentum (Brandon, The Fall of Yerusalem, S. 54 ff.; Brandon, The Trial of Gesus, S. 62 f. - Es ist wahr, dass die heidenchristliche Kirche eine andere ist als die palästinische judenchristliche. Aber der Unterschied muss anders bewertet werden, als Brandon es tut. Richtig beurteilt Hengel, S. 316: ,Das NichtEintreten der erhofften Heilszeit brachte für das Judentum keine geringere Erschütterung als für die ebenfalls durch die Naherwartung bestimmte frühchristliche Gemeinde. Die Entwicklung zum Rabbinat läuft parallel zur Herausbildung der frühkatolischen Kirche." Während Brandon mit einem vollständigen Bruch des Heidenchristentums mit dem palästinischen Judenchristentum und einem radikalen Neuanfang rechnet, so impliziert Hengels Betrachtung eine nicht zu unterschätzende Kontinuität zwischen den beiden Kirchen. Siehe unsere Auseinandersetzung mit Brandon in der Fortsetzung). Die Urkunden dieser neuen Religion sind die Evangelien und die Apostelgeschichte. Jetzt entsteht das Bild des ,pazifistischen Christus“ (the pacific Christ, Brandon, Fesus and the Zealots, S. 320). Die Darstellung seiner Geschichte ist gänzlich antijüdisch und prorömisch (Brandon, The Fall of $\mathscr{Y}$ erusalem, 
ren. Insofern Jesus selbst in messianischen Kategorien gedacht hat, so hat er an die Menschensohnsvorstellung angeknüpft, wie wir sie vor allem durch das äth. Henoch kennen. Aber es ist dabei mit Händen zu greifen, dass Jesus diese Vorstellung mit einem neuen Inhalt gefüllt hat teils im Anschluss an Jes. 53 und teils gemäss seinen eigenen Worten, er sei gekommen um die Verlorenen und Irregegangenen zu retten. Seine Mission ist geistig-religiös, und es besteht kein Grund für die Behauptung, er habe Pläne gehabt, sich für das populäre jüdische Messiasprogramm einzusetzen. Jesus war ein Ketzer. So wurde er auch in der jüdischen Tradition angesehen. Er hat eine

S. I $85 \mathrm{ff}$; Brandon, The Trial of $\mathscr{Y} e s u s$, S. $65 \mathrm{ff}$.). Die Bücher Brandons sind von bedeutendem Interesse. $\mathrm{Zu}$ ihrem bleibenden Wert gehört die Darstellung der jüdischen Geschichte in den Jahren 6-70 p. Chr. und der zelotischen Bewegung. Viele Einzelheiten dieser dunklen Periode und damit auch der ältesten Geschichte des Christentums werden durch scharfsinnige Beobachtungen in neues Licht gerückt. Nicht zum mindesten wird die Problematik der Passionsgeschichte mit bewunderungswerter Energie aufs neue und nach neuen Einfallswinkeln geschärft. Aber um seine monolithische These durchzuführen wird der Autor gezwungen, gegen die Gesetze der sauberen Quellenkritik zu verstossen. Er macht einen titanischen Versuch, die Geschichte des Werdens des Christentums radikal umzudeuten. Das geschieht auf Kosten sichergestellter Resultate oder bis zur Evidenz grenzender Wahrscheinlichkeit. Es geht nicht an, die ganze in den Evangelien aufbewahrte Jesustradition als eine späte, heidenchristliche, völlig unhistorische Tendenzgeschichte wegzuerklären. Brandon beabsichtigt eine Relronstruktion der Jesusgeschichte, er sucht wie viele vor ihm den historischen Jesus. Aber eine aufmerksame Musterung seiner Rekonstruktion macht deutlich, dass es sich um eine Konstruktion handelt, die auf zu viele Vermutungen baut um bestehen zu können. Dieser Konstruktion muss entgegengehalten werden: Die Jesusbewegung war kein Gegenstück zum militanten Zelotismus (,,a kind of para-Zelot movement", Brandon, Fesus and the Zealots, S. 201). Es sei jedoch zugestanden, dass Jesus als ein wahrer Jude dem Schicksal des auserwählten Volkes nicht gleichgültig gegenüberstand. Vgl. Gwyn Griffiths, S. $358 \mathrm{ff}$. Ich zitiere: „It need hardly be added that the superscription fastened to his own cross included the significant words THE KING OF THE JEWS, which are present, in spite of other variants, in each Gospel record, and/constitute irrefutable evidence of the resistance to Rome which was a part of the attitude of Jesus although he manifestly rejected armed rebellion ... Resistance to Rome, one must agree, was a vital part of Christ's Messiahship, but the distinctive thing about his resistance, which he also inculcated upon his disciples, was that it was non-violent and extended the principle of love to include even the Roman oppressors" (S. $3^{6}$ I f.). Ferner: ,It seems likely that the interpretation of the Gospels has, in general, underestimated the all-pervading force of this nationalism ... Spiritual and non-violent resistance was, nevertheless, an essential part of the Messianic ideal as pursued by Jesus" (S. 363). Dies ist schon viel gesagt, vielleicht $z u$ viel. Stärker und sicher nicht ohne Grund wird der Gegensatz Jesu zu den Zeloten von Hengel, S. 345, und Flusser, S. 8I ff. betont. 
radikale religiöse Botschaft gepredigt, den Gegensatz der "Normalreligion“. Die in den Evangelien aufbewahrte Tradition zeugt unwidersprechlich davon. Der Zelotismus war auch religiös, aber seine Religion war ganz anderer Art, sie war eine politische Religion. Er hatte den heiligen Krieg gegen die Römer gepredigt. Jesus trat mit seiner Botschaft am Vorabend grosser Katastrophen auf. Diese zeitliche Koinzidenz der durch Jesus geschaffenen Bewegung und des Zelotismus erklärt wenigstens zum Teil die Kreuzigung Jesu zusammen mit zwei Zeloten, eine unermessliche Tragödie der jüdischen Geschichte.

Wir stossen im Neuen Testament auf eine ganze Flora der mit dem antiken Staatswesen zusammengehörenden Terminologie. Einige Beispiele seien zum Beleg angeführt. Das Hirnmelreich wird mit einem König (anthropos basileus) und seinem Handeln verglichen, Matth. I8: 28, 23 : I ff. Die Begriffe polis, politeuma, patris, basileia kommen oft in figurativer Anwendung vor. Gott ist pantokrator, Christus ist basileus basileon, König der Könige und kyrios kyrion, der Herr der Herren (aus der orientalischen Hofsprache' ${ }^{1}$ ). Der Menschensohn wird im Gleichnis vom jüngsten Gericht König genannt (Matth. 25:3 I ff.). Er ist König im tausendjährigen Reich (Off. 20: 4). In der apokalyptischen Spekulation wird die endgültige Begleichung mit der satanischen Macht als eine Schlacht zwischen dem himmlischen von Christus angeführten Heer und den gesammelten Truppen irdischer Kriegsobersten geschildert. Die Endvision der Apokalypse hat zum Thema das himmlische Jerusalem, das auf die Erde niederkommt. Politisch? Nein! Gegen den Mythus vom Weltreich wird der Antimythus vom Reich, das nicht von dieser Welt ist, gestellt.

Wie wir aus der jüngsten Geschichte erfahren haben, wird eine geistige Grösse wie die des Christentums von einer totalitären Staatsideologie als politisch gefährlich und staatsfeindlich gestempelt. Eben das A-politische wird von den politischen Ideologien als Anti-politisch aufgefasst. Das ist eigentlich selbstverständlich, wenn z. B. ein religiöses System eine andere Auffassung vom Menschen vertritt als die der Staatsideologie. Die Aussage Jesu „Mein Reich ist nicht von dieser Welt“ ist ein a-politisches Wort, aber

\footnotetext{
${ }^{1}$ So auch in der jüdischen Gebetssprache von Gott. Er ist „König der Könige“, Hengel, S. 100.
} 
sie kann ein anti-politisches Wort werden und damit auch aus dem Gesichtspunkt der politischen Gesellschaft als politisch gefährlich aufgefasst werden.

Jesus war kein politischer Revolutionär, er hat nicht Gewalt proklamiert, sondern das Gegenteil. Wenn Revolutionären sich auf ihn berufen um mit Gewalt die Gesellschaft zu stürzen, so ist das die denkbar gröbste Verdrehung von Tatsachen. Das Evangelium ist a-politisch aber keineswegs darum im Leben der Gesellschaft ein harmloses Neutrum. Es bietet kein Programm für Zeloten, aber es kann von seinen Bekennern das Martyrium verlangen ${ }^{1}$. Probatum est!

${ }^{1}$ Vgl. hierzu W. A. Beardslee, „New Testament Perspectives on Revolution as a Theological Problem“, The fournal of Religion, 51, I97I, S. I 5 ff.; E. Grässer, ,'Der politisch gekreuzigte Christus'. Kritische Bemerkungen zu einer politischen Hermeneutik des Evangeliums", Zeitschrift für die neutestamentliche Wissenschaft und die Kunde der älteren Kirche, 62, 1971, S. 266 ff; W. Schrage, Die Christen und der Staat nach dem Neuen Testament, Gütersloh I97I. 